\title{
Lentes de contato gelatinosas tóricas
}

\author{
Toric soft contact lenses
}

Cleusa Coral-Ghanem

\section{INTRODUÇÃO}

As lentes de contato gelatinosas (LCG) esféricas, diferente das lentes de contato (LC) rígidas e rígidas gás-permeáveis (RGP), não mascaram o astigmatismo corneal por serem moles e flexíveis e se amoldarem à córnea subjacente. Para corrigir o astigmatismo, corneal e/ou residual, foram desenvolvidas as LCG tóricas. As primeiras LCG tóricas tornaram-se disponíveis em 1975. Holden, discutindo o critério para a prescrição de LC tóricas verificou que, numa população superior a 20.000 usuários de LC, 45\% eram portadores de astigmatismo superior a 0,75 dioptrias (D) (média de $0,83 \mathrm{D}$ ). Desses, $95 \%$ apresentavam 1,9 D de astigmatismo com-a-regra, $0,25 \mathrm{D}$ contra-aregra e menos de $2 \%$ eram portadores de astigmatismo maior do que $3 \mathrm{D}^{(1)}$.

As técnicas utilizadas para a estabilização de LCG tóricas, até a década de 80 , eram o prisma de lastro e a truncagem que as tornavam espessas e desconfortáveis. A partir daí, principalmente pelo desenvolvimento da técnica de estabilização dinâmica que, conforme referência ${ }^{(2)}$, foi iniciada por Fanti em 1975, as LC tornaram-se mais leves e possibilitaram o uso prolongado (UP). Além disso, esse tipo de desenho, associado ou não a outras técnicas de estabilização, permitiu que as LC alcançassem orientação mais estável do eixo e melhor reprodutibilidade.

Na última década, foram desenvolvidos desenhos de LCG tóricas capazes de corrigir quase todos os graus de astigmatismo regular com resultados visuais bastante satisfatórios. Apesar disso, o número de adaptações é ainda muito pequeno em relação às reais possibilidades. A maioria dos portadores de astigmatismo, bons candidatos para LCG tóricas, continuam com óculos porque persiste na população o conceito antigo de que o astigmatismo só pode ser corrigido com LC rígidas.

O objetivo deste artigo é apresentar uma revisão atualizada da adaptação de LCG tóricas. O trabalho está dividido nos seguintes itens: métodos de fabricação; desenhos de LCG tóricas; técnicas de estabilização; indicação de LC conforme o tipo de astigmatismo; critérios básicos para a indicação; fato-

Endereço para correspondência: Hospital de Olhos Sadalla Amin Ghanem - R. Abdon Batista, 172 - Centro - Joinville (SC) CEP 89201-010. E-mail: coral.joi@zaz.com.br res que interferem na estabilização; métodos de adaptação; técnica e avaliação da adaptação; características de LCG tórica bem adaptada, apertada e plana; principais causas de distúrbios visuais em usuários de LCG tórica; dicas de adaptação e de avaliação; tipos de LCG tóricas.

\section{MÉTODOS DE FABRICAÇÃO}

São três os mais utilizados:

- fresagem;

- reprodução, que é uma forma especial de torneamento voltada à produção de superfícies tóricas;

- associação de torneamento mais moldagem (superfície anterior torneada e posterior moldada).

\section{DESENHOS DE LCG TÓRICAS}

Para a escolha do desenho, leva-se em conta a refração, a toricidade da córnea, o método de estabilização da LC, o desempenho fisiológico e as considerações relativas à fabricação.

Os dois tipos básicos de desenho apresentam:

- superfície posterior tórica com superfície frontal esférica;

- superfície posterior esférica com superfície frontal tórica.

A fabricação do primeiro é mais complexa por requerer maior número de etapas, entretanto apresenta maior estabilidade em cilindros altos.

Com os métodos atuais de fabricação, nenhum deles tem vantagens especiais quando se trata de LC com cilindros de 0,75 a 2,50 D. A LCG tórica assume a configuração de bitórica quando colocada sobre uma córnea tórica. A toricidade é transferida de uma superfície para a outra.

\section{Superficie Posterior Tórica com Superfície Frontal Esférica}

É desenhada de forma a encaixar-se sobre a córnea tórica, neutralizando o astigmatismo e auxiliando a estabilização da LC. O astigmatismo lenticular não é, teoricamente, corrigível pelo uso desse tipo de desenho. Clinicamente, entretanto, resultados razoáveis podem ser obtidos.

Indica-se para altos astigmatismos corneais, principalmente se o componente esférico for menor do que o cilíndrico. 


\section{Superfície Posterior Esférica ou Asférica com Superfície Frontal Tórica}

Este desenho é indicado quando o astigmatismo for residual ou corneal até 2,00 D. Assume característica bitórica quando colocada sobre a córnea tórica. A estabilização da LC sobre a córnea pode ser feita por meio de prisma de lastro, truncagem ou pelo método de estabilização dinâmica. Sua espessura e geometria impõem um limite máximo de cilindro em torno de 4,50 D.

\section{TÉCNICAS DE EST ABILIZAÇ̃̃̃O}

Para evitar a rotação da LC e manter a acuidade visual AV estável, podem ser utilizadas várias técnicas:

- Superfície posterior tórica;

- Prisma de lastro;

- Peri-lastro;

- Desenho truncado;

- Estabilização dinâmica (desenho chanfrado);

- Lenticulação excêntrica;

- Combinação das técnicas acima citadas.

Independentemente do método de estabilização escolhido, a orientação estável do eixo é o resultado do diferencial da espessura ao longo da porção superior, central e inferior da LC.

\section{Superfície Posterior Tórica}

A estabilização com este tipo de desenho, conforme já mencionado, é conseguida pelo efeito de encaixe da LC sobre a córnea tórica, como se fosse uma luva.

Tanto em superfície tórica posterior como em anterior, o desenho da LC deve incorporar o prisma estabilizador apenas na sua área periférica. Uma zona óptica livre de prisma permite que a LC seja produzida mais fina. Apresenta a vantagem adicional de não introduzir o desequilíbrio vertical quando apenas um dos olhos necessita correção tórica.

\section{Prisma de Lastro (Figura 1A)}

O princípio básico do prisma de lastro é exercer peso. Junto com o fator gravidade, auxilia no posicionamento da LC, puxando-a para baixo após o piscar. Utiliza-se um prisma de $1,00 \mathrm{a} 1,50 \mathrm{D}$, de base inferior, cujo centro está localizado às $6 \mathrm{~h}$. A espessura aumentada, associada ao pouco movimento da LC, pode causar edema corneal localizado, além de provocar desconforto em pacientes com pálpebras sensíveis.

\section{Peri-lastro}

A estabilização é conseguida pela remoção de um "carrier" lenticular alto negativo da porção superior da LC. Essa técnica reduz a interação pálpebra-borda da LC e produz um lastro na porção inferior da mesma. Na prática, é semelhante ao prisma de lastro, exceto que, no peri-lastro, todo o prisma está fora da região da zona óptica.

\section{Desenho Truncado (Figura 1B)}

Caracteriza-se pela remoção, em corte horizontal, de 0,50 a $1,50 \mathrm{~mm}$ de largura na borda inferior, durante o processo de fabricação. Alguns fabricantes utilizam o desenho truncado também na porção superior da LCG. Quanto maior o diâmetro, maior poderá ser a parte seccionada. A finalidade da truncagem é apoiar a borda cortada na borda palpebral inferior. Com o propósito de melhorar a estabilização, costuma-se associar prisma de lastro de base inferior, que tem a desvantagem de aumentar a espessura e o desconforto.

Para o sucesso do uso da LCG truncada, o posicionamento da borda palpebral inferior deve estar no limbo inferior.

\section{Estabilização Dinâmica (bordas chanfradas) (Figura 1C)}

É alcançada pelo afilamento da superfície anterior, em sua parte inferior (simples "slab-off") ou superior e inferior (duplo "slab-off"), com o objetivo de reduzir a espessura, encaixar a LC sob as pálpebras e proporcionar conforto. As zonas afiladas no topo e na base da LC fazem com que o centro mais espesso se posicione entre elas, promovendo estabilização dinâmica. Com essa técnica, a toricidade da LC fica limitada à porção central. O diâmetro da zona óptica costuma ser de $8 \mathrm{~mm}$, situando-se dentro da abertura palpebral.

A técnica de estabilização dinâmica é uma das mais utilizadas, por ser facilmente reprodutível e poder ser associada a outros métodos.

\section{Lenticulação Excêntrica (Figura 1D)}

Atualmente, muitos laboratórios utilizam a lenticulação excêntrica, especialmente na construção da LC tórica frontal, na qual a espessura da borda pode ter variação significativa. A remoção do excesso de material da superfície anterior, semelhante ao peri-lastro, reduz a espessura da borda, aumenta a estabilidade, melhora o conforto e minimiza a compressão na conjuntiva escleral. A espessura torna-se quase igual em torno da periferia da LC e o prisma permanece nos $2 / 3$ centrais dela. A estabilidade proporcionada por esse desenho é mais evidente em correções de cilindro oblíquo, quando a abertura da margem palpebral encontrando primeiro a parte mais espessa da LC, num ângulo oblíquo, costuma provocar deslocamento torsional.

\section{CRITÉRIOS BÁSICOS PARA A INDICAC̄̃̃O DE LCG TÓRICAS}

O astigmatismo da córnea é mensurável por meio do ceratômetro ou da videoceratoscopia. Sua comparação com a refração dos óculos demonstra a possibilidade de haver astigmatismo residual.

Para prescrever LCG tórica, deve-se avaliar cada candidato individualmente, considerando-se os fatores adiante:

\section{Quantidade e Tipo de Astigmatismo}

Indica-se $\mathrm{LCG}$ tórica em astigmatismo:

- regular, igual a, ou maior do que 1,00 D; 


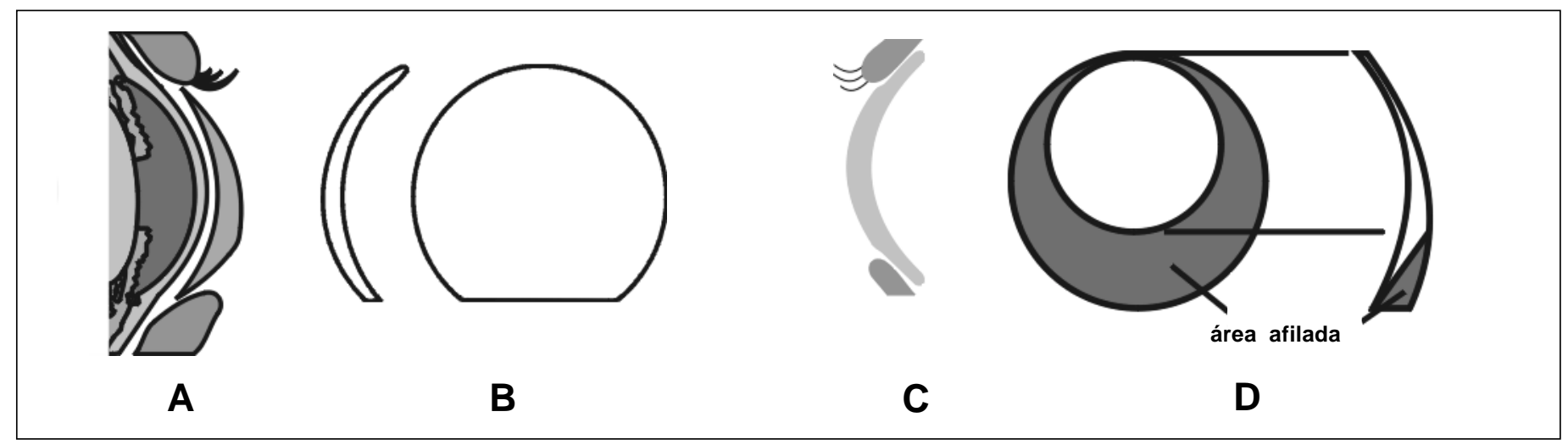

Figura 1 - Desenhos de lentes de contato: A) com prisma de lastro; B) truncada; C) com bordas chanfradas (perfil); D) com lenticulação excêntrica

- regular de $0,75 \mathrm{D}$, quando representar um terço ou mais do erro refrativo total;

- lenticular (residual), encontrado freqüentemente em associação a córneas esféricas ou com moderado astigmatismo contra-a-regra;

- irregular e ceratocone, quando o paciente não tolerar LC RGP, se fornecer AV satisfatória.

Evita-se em astigmatismo:

- irregular, sempre que for possível adaptar RGP;

- associado à LC bifocal/multifocal gelatinosa;

- pequeno, associado à ambliopia;

- abaixo de 1,00 D, quando o paciente estiver satisfeito com LCG esférica, especialmente se fizer apenas uso ocasional ou se estiver preocupado com o custo da LCG tórica.

\section{Eixo do Astigmatismo}

Se o eixo do meridiano mais plano da córnea for diferente do eixo do cilindro dos óculos, é recomendável adaptar LC RGP, que tem o poder de mascarar o cilindro cruzado resultante. O mesmo não acontece com LCG tórica, que tende a se acomodar sobre o meridiano primário da córnea.

O astigmatismo miópico oblíquo contra-a-regra, não corrigido, causa mais instabilidade visual e astenopia do que o astigmatismo com-a-regra.

\section{Avaliação das Necessidades Visuais}

Quanto maior a necessidade visual do paciente nas suas tarefas diárias, menor a quantidade de astigmatismo que ele tolera. Por exemplo, um digitador pode necessitar a correção de um cilindro tão pequeno quanto $0,50 \mathrm{D}$ para possibilitar seu trabalho. Por outro lado, uma pessoa sem tarefas visuais específicas pode sentir-se satisfeita com um astigmatismo residual maior do que 1,00 D, contanto que o componente esférico de seu erro refrativo seja corrigido. Esse fato é freqüente em pessoas que utilizam LCG para a prática de esportes.

Présbitas jovens requerem especial consideração porque $o$ eixo da LC desvia no olhar para baixo, podendo afetar a AV e fatigar o sistema de acomodação.
Alguns fabricantes (Califórnia Optics, Ocu-Ease Optical, United Contact Lens e Westcon Contact Lens) estão disponibilizando, nos Estados Unidos, as LCG tóricas bifocais.

\section{Predominância Ocular}

Pacientes podem tolerar cilindro não corrigido de até $1,50 \mathrm{D}$ no seu olho não-dominante, mas ao mesmo tempo exigem que um cilindro tão pequeno quanto $0,50 \mathrm{D}$ seja corrigido no olho dominante. Astigmatismo residual é facilmente tolerado por pessoas portadoras de ametropias altas e AV abaixo do normal.

\section{Considerações Ópticas}

Para calcular uma LCG tórica não é necessário considerar a lente lacrimal. Todo o astigmatismo ocular é, normalmente, corrigido pela incorporação do cilindro no seu desenho.

Consegue-se AV semelhante à fornecida pelos óculos quando o astigmatismo se apresenta em posição próxima ao meridiano vertical ou ao horizontal. Eixos oblíquos freqüentemente fornecem resultados visuais piores do que os óculos. Costumam ter melhor correção com LC rígida esférica ou asférica.

Astigmatismo maior do que 3,00 D geralmente apresenta melhor resultado visual com LC RGP tórica, ao invés de LCG tórica.

\section{Anatomia e Tônus Palpebral}

O deslocamento da LC e sua rotação têm como causa principal a dinâmica das pálpebras. Fissuras palpebrais pequenas ou pálpebras muito apertadas costumam provocar pressão excessiva sobre a LC, alterando sua estabilidade rotacional. No entanto, uma abertura palpebral muito ampla, quando associada a um padrão de piscamento incompleto, facilita a desidratação da LC e a formação de depósitos. Os sintomas são mais percebidos pelos pacientes portadores de alterações do filme lacrimal. A pálpebra ideal deve ter tensão normal e uma abertura relativamente ampla. Além disso, não deve apresentar elevação de tecido conjuntival ${ }^{(3)}$. 


\section{FATORES QUUE INTERFEREM NA EST ABILIZAÇÃO DA LCG}

- Ação das pálpebras;

- Desenho da LC, que depende do erro refrativo e da técnica de estabilização;

- Efeito da gravidade;

- Espessura;

- Relação da adaptação da LC sobre a córnea.

A espessura é determinada, particularmente, pelo eixo e grau do astigmatismo. Para as LCG tóricas que incorporam estabilização dinâmica, a influência rotacional é maior nas LC com cilindros em eixos oblíquos (entre $30^{\circ}$ e $60^{\circ}$ ou entre $120^{\circ} \mathrm{e}$ $\left.150^{\circ}\right)^{(4)}$. Conforme esse autor, o principal fator que afeta a rotação é o ponto inicial de contato entre a pálpebra superior e o meridiano mais espesso da LC. Nas LCG tóricas de eixo oblíquo, isso implica efeitos rotacionais importantes, uma vez que o contato com a pálpebra superior sempre afetará uma margem do meridiano mais espesso antes da outra. Quando a pálpebra superior desce, força a LC para baixo nesse primeiro ponto de contato, determinando a sua rotação numa certa direção.

\section{MÉTODO DE ADAPTAÇÃO EMPÍRICO}

Dispensa-se o teste com LC de prova e encomenda-se a LCG tórica pela ceratometria e refração, na forma de cilindro negativo, levando em conta a orientação do fabricante com o qual se trabalha.

- Grau Esférico - usar o da refração dos óculos, corrigindo a distância ao vértice acima de $4 \mathrm{D}$.

- Grau Cilíndrico - solicitar igual ou menor do que o cilindro da refração.

- Curva Base (CB) - utilizar a ceratometria e as opções fornecidas pelo fabricante.

- Eixo do cilindro:

OD - Somar $5-10^{\circ}$ do eixo do cilindro da refração;

OE - Subtrair $5-10^{\circ}$ do eixo do cilindro da refração.

Com esse método não se prevêem as alterações de posicionamento do eixo do cilindro causadas pela flexibilidade do material e pelo movimento das pálpebras.

\section{MÉTODO DE ADAPTAÇÃO COM LCG TÓRICA DE TESTE}

Os princípios de adaptação para LCG tóricas são muito semelhantes aos da LCG esféricas. A diferença está no movimento que deve ser menor nas LCG tóricas destinadas a corrigir graus altos de astigmatismo.

\section{Técnica de Adaptação}

Para iniciar o teste, seleciona-se da caixa de prova a LC com grau esférico, grau cilíndrico e eixo mais próximos da refração do paciente. Utiliza-se curva base (CB) de acordo com os parâmetros ceratométricos. Alguns tipos de LC estão dis- poníveis apenas na CB média. Aguardar de 20 a 30 minutos para sua estabilização e examinar à lâmpada de fenda. Esse tempo é necessário para que haja a troca da solução de manutenção pela lágrima do paciente, pois pequenas variações na tonicidade e pH das soluções em relação a composição individual da lágrima podem alterar as características de adaptação.

\section{Avaliação da Adaptação}

\section{- Movimento}

O movimento vertical, durante o piscar deve ser de, aproximadamente, 0,25 a $0,50 \mathrm{~mm}$ quando o olho está em posição primária. No olhar para cima ou para os lados, um movimento de $0,5 \mathrm{~mm}$ é suficiente para liberar os debris da interface córnea-LC, sem causar instabilidade do eixo.

\section{- Diâmetro}

O diâmetro total da LC influencia sua centralização e estabilidade. Prefere-se utilizar diâmetro grande, pois representa uma área maior disponível para as zonas de estabilização na periferia da $\mathrm{LC}$.

\section{- Rotação}

A estabilidade é afetada pela ação das pálpebras. A pálpebra superior, ao se fechar, desloca a LC no sentido oblíquo, fazendo-a girar, deslocando-a para o lado nasal ou para o temporal. A influência da pálpebra inferior só é observada quando se utiliza LC tórica truncada.

Para auxiliar na avaliação da rotação do eixo, alguns fabricantes utilizam marcas a laser nos meridianos de $90^{\circ}$ e $180^{\circ}$; outros, o sistema de relógio através de marcas às $3,6 \mathrm{e} 9$ horas, com distância de $30^{\circ}$ entre cada hora. Em algumas LC essas marcas estão a $10^{\circ}, 15^{\circ}$ ou a $20^{\circ}$ umas das outras (Figura 2).

Essas marcas servem para orientar a quantidade de rotação da LC em cada paciente, mas não significa a localização do eixo do cilindro.

A análise da rotação para a determinação do eixo a ser solicitado é realizada, à lâmpada de fenda, 20 min, ou mais, após a colocação das LC.

A LC definitiva, após um período de adaptação, deverá apresentar rotação idêntica à LC de prova.

\section{Eixo sem rotação}

Analisando-se, por exemplo, a Soflens Toric (B\&L), se as marcas de 5,6 e $7 \mathrm{~h}$ coincidirem com as de um relógio, nenhuma compensação do eixo da refração será necessária.

Eixo do cilindro da $\mathrm{LC}$ a ser solicitada $=$ Eixo da receita dos óculos (Figura 3A).

\section{Rotação no sentido horário}

Se a LC de teste apresentar rotação no sentido horário, adicionar o total da rotação ao eixo da refração.

Eixo da LC a ser adaptada = Eixo da receita do óculos + graus de rotação apresentados pela LC de prova (Figura 3B). Exemplo:

Rx dos óculos: $-3,50-1,50 \times 100^{\circ}$

LC de teste: $-3,50-1,50 \times 90^{\circ}$ 


\begin{tabular}{|c|c|c|c|c|c|c|c|}
\hline $\begin{array}{c}\text { OPTIFIT } \\
\text { D2 ou D3 } \\
\text { Separação de } \\
20^{\circ}\end{array}$ & $\begin{array}{c}\text { Hydrocurve } 3 \\
\text { (WJ) } \\
\text { Optima Toric } \\
\text { e Soflens } 66 \\
\text { Toric (B\&L) } \\
\text { Separação } \\
\text { de } 30^{\circ}\end{array}$ & $\begin{array}{c}\text { Hydrasoft e } \\
\text { Preference } \\
\text { Toric (Cooper } \\
\text { Vision) } \\
\text { Sunsoft } \\
\text { (Sunsoft) }\end{array}$ & $\begin{array}{c}\text { Flexlens } \\
\text { Toric } \\
\text { (Flexlens) }\end{array}$ & $\begin{array}{l}\text { Focus Toric } \\
\text { (ClBA) } \\
\text { Proclear } \\
\text { Toric } \\
\text { (Biocompatibl } \\
\text { es) }\end{array}$ & $\begin{array}{l}\text { Hydron Ultra } \\
T \text { (Ocular } \\
\text { Sciences) } \\
\text { Separação } \\
\text { de } 15^{\circ}\end{array}$ & $\begin{array}{l}\text { Fresh Look } \\
\text { Toric (WJ) }\end{array}$ & $\begin{array}{l}\text { Acuvue Toric } \\
\text { (Johson\& } \\
\text { Johson) } \\
\text { Separação } \\
\text { de } 10^{\circ}\end{array}$ \\
\hline
\end{tabular}

Figura 2 - Marcas para orientar a rotação de LC

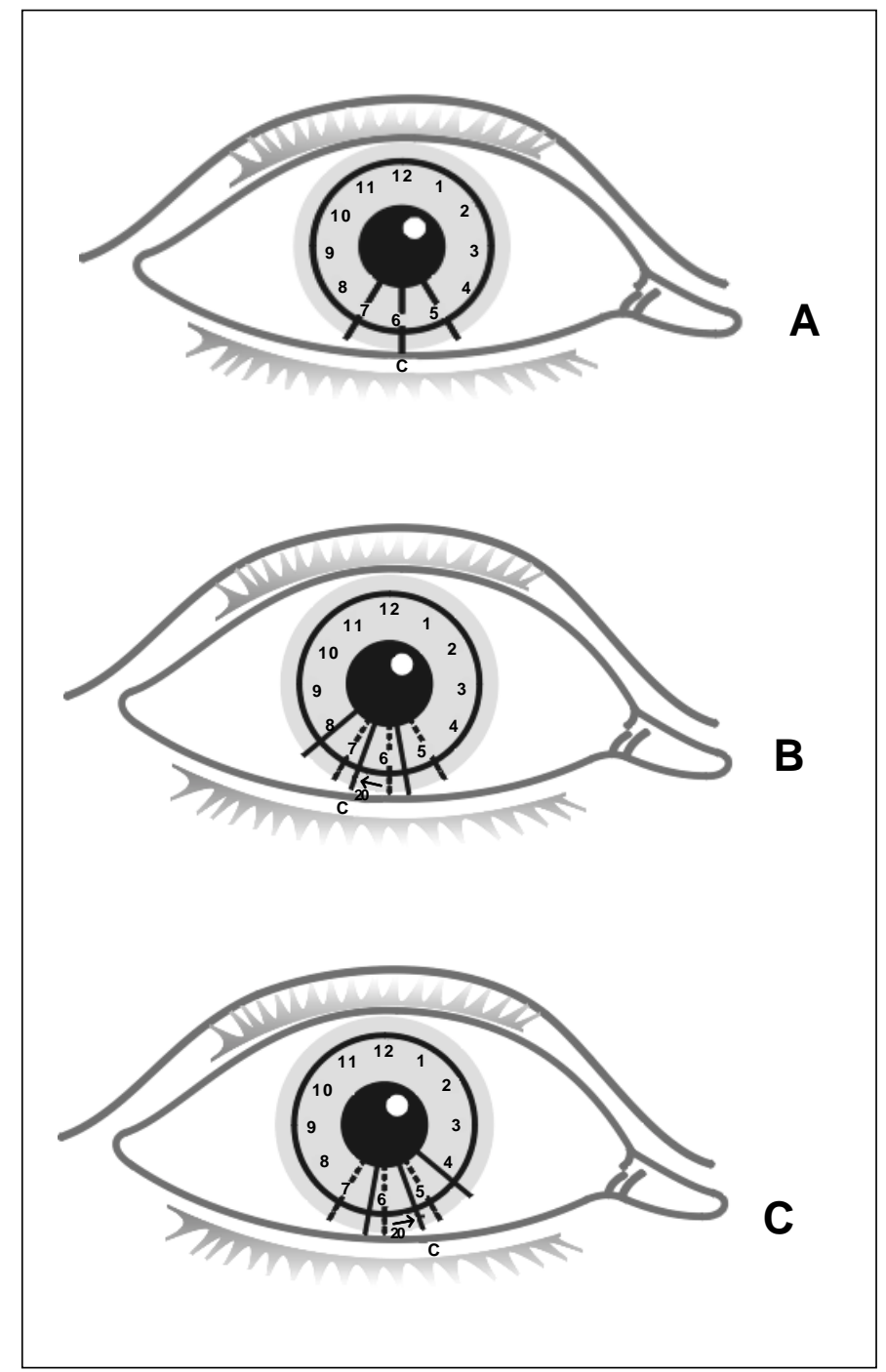

Figura 3 - A) LC centralizada; B) LC deslocada $20^{\circ}$ no sentido horário; C) LC deslocada $20^{\circ}$ no sentido anti-horário
Localização: marca das $6 \mathrm{~h}$ desviada $20^{\circ}$ no sentido horário. LC a solicitar: $-3,50-1,50 \times 120^{\circ}$

\section{Rotação no sentido anti-horário}

Nesse caso, deve-se subtrair o total da rotação do eixo da refração.

Eixo da LC a pedir = Eixo da receita dos óculos - graus de rotação apresentados pela LC de prova (Figura 3C).

Exemplo:

Rx dos óculos: $-3,50-1,50 \times 100^{\circ}$

LC de teste: $-3,50-1,50 \times 90^{\circ}$

Localização: marca das $6 \mathrm{~h}$ desviada $20^{\circ}$ no sentido antihorário.

LC a solicitar: $-3,50-1,50 \times 80^{\circ}$

\section{- Determinando o Grau}

É indispensável que a refração seja feita corretamente. Em indivíduos com altas ametropias (graus superiores a $+4,00 \mathrm{e}$ - 4,00 D), ajusta-se o componente esférico da prescrição dos óculos, considerando a distância que as lentes ficam do vértice das córneas. Essa correção também é necessária na prescrição de LCG tóricas visto que, atualmente, estão disponíveis para erros refrativos altos.

Se um paciente tiver um cilindro de $-2,00 \mathrm{D}$ e a LC que se deseja adaptar só está disponível em -1,75 e -2,25, o oftalmologista deve optar pelo grau mais baixo. Assim, quando a LC girar fora do eixo, irá provocar menos astigmatismo residual.

\section{CARACTERÍSTICAS DE UMA LCG TÓRICA BEM ADAPTADA}

- Boa centralização e estabilidade rotacional.

- Cobertura corneal completa, sem contato da borda com o limbo.

- Conforto em todas as posições do olhar.

- Movimento suficiente para permitir troca de lágrimas sob a LC durante o piscar, em posição primária ou superior, ou quando se faz o "push-up test"(5). 
- Rápido retorno à posição original do eixo após o piscar.

- Visão estável em todas as posições do olhar.

\section{Características de uma LC Apertada}

- Boa centralização.

- Bom conforto.

- Possível deslocamento estável do eixo.

- Pouco ou nenhum movimento durante o piscar, em posição primária ou no olhar para cima.

- Visão borrada entre um piscar e outro.

LC muito apertada causa turvação visual, que melhora após a piscada. Entretanto, se estiver apenas um pouco apertada, a visão não varia com o piscar e o conforto inicial é bom. Para diferenciar uma LC ligeiramente apertada de uma bem adaptada, pede-se ao paciente que olhe para cima. Uma LC bem adaptada tende a descer de 0,5 a $1 \mathrm{~mm}$, enquanto uma LC apertada permanece imóvel. Pode-se fazer o "push-up test" ou, então, girar gentilmente a LC com o dedo, em aproximadamente $45^{\circ}$, para o lado temporal. Após 5 a 10 piscadas (não mais do que 15 segundos), ela deve retornar à sua posição inicial.

Quando a LC se comporta desse modo deve ser readaptada com uma CB mais plana ou com outro desenho.

\section{Características de uma LC Plana}

- Descentralização.

- Discreto desconforto.

- Levantamento da borda.

- Movimento excessivo durante o piscar, em posição primária ou no olhar para cima.

- Visão borrada imediatamente após o piscar.

LC muito plana provoca visão nublada após o piscar e desce mais do que $1 \mathrm{~mm}$ no olhar para cima. O giro é maior do que $5^{\circ}$, oscilando na posição de orientação entre as piscadas. Pode apresentar borda inferior afastada da esclera, provocando desconforto e irritação ocular, além de visão flutuante.

Quando a LC se comporta desse modo deve ser readaptada com uma CB mais apertada ou com outro desenho.

\section{PRINCIPAIS CAUSAS DE DISTÚRBIOS VISUAIS}

\section{Rotação da LC}

Pode provocar distúrbio visual permanente ou temporário após cada piscar. Pacientes que apresentam astigmatismo num eixo oblíquo são mais propensos à rotação da $\mathrm{LC}$ do que aqueles que apresentam astigmatismo com-a-regra e contra-aregra. Um efeito de cilindro cruzado oblíquo, resultante do mau alinhamento entre o eixo do cilindro refrativo e o da LC tórica, provoca distúrbios na AV. A visão de perto pode ser comprometida pela rotação da LCG tórica com a convergência do olho, resultado da ciclo-rotação do globo ocular. Esse problema é ainda mais acentuado em pacientes présbitas que fazem monovisão, usando LCG tórica.

O exame à lâmpada de fenda determina se a LC apresenta centralização e movimento adequados, ou se ocorre oscilação excessiva após o piscar. O movimento excessivo pode exigir CB mais apertada ou um desenho diferente para reduzir a rotação e melhorar a AV.

A refração realizada sobre a LC em uso ou sobre a LC diagnóstica pode ser útil para determinar o grau e o eixo corretos.

\section{SUGESTÕES PARA O SUCESSO DA ADAPTAÇÃO}

- Aguardar pelo menos 20 minutos para avaliar a LCG tórica de teste.

- Avaliar a quantidade de rotação que, em graus altos, não deve ser maior do que $5^{\circ} \mathrm{e}$, nos baixos, pode ser de até $10^{\circ}$.

- Corrigir a distância ao vértice em cada meridiano, nos graus superiores a 4,00 D.

- Fazer refração cuidadosa para obter a melhor AV possível.

- Fazer o teste para predizer o sucesso de adaptação da LCG tórica (Becherer Twist). Quando se tem a melhor refração subjetiva no refrator, gira-se o eixo do cilindro até o paciente perder a nitidez da imagem na tabela de AV. Nesse momento, avalia-se o desvio:

- Maior do que 20 graus em cada direção, independentemente da localização do eixo ou da quantidade de cilindro, prevê um sucesso de adaptação $>90 \%$ com a primeiraLC;

- Igual a 15 graus, o êxito será de aproximadamente $90 \%$ com duas LC;

- Igual a 10 graus, será de 70\%, utilizando-se três LC;

- Menor do que 5 graus, o sucesso dependerá da quantidade de redução de nitidez que o paciente está disposto a aceitar.

- Informar ao paciente que é comum a necessidade de várias trocas antes da LC definitiva.

- Optar pelo desenho que apresente a melhor estabilidade rotacional.

- Usar o menor grau cilíndrico que forneça AV aceitável para que não haja sintomas quando a LC sofrer pequenas rotações.

\section{AVALIAÇÃO DA LCG TÓRICA SOLICITADA}

- Se a visão alcançada com a LC for inferior à proporcionada pelos óculos, é recomendável reavaliar o paciente após uma semana de uso, antes de solicitar a troca da LC.

- Se a visão for insatisfatória e a sobre-refração da LC não alterar o resultado, recomenda-se modificar a CB. Fisicamente, a LC no olho poderá parecer a mesma, mas as diferentes curvas da sua face posterior criam um outro sistema óptico, podendo fornecer uma melhor AV.

- Se uma LC não apresenta a estabilidade ideal, mas o paciente está satisfeito, é recomendável não perseguir a 
perfeição. Como há poucas opções de $\mathrm{CB}$ e diâmetros disponíveis para LCG tóricas, deve-se determinar um nível de tolerância aceitável.

\section{TIPOS DE LCG TÓRICAS}

\section{Convencionais - Troca Anual}

Denomina-se LCG tóricas convencionais aquelas que são programadas para troca anual. Podem ser fabricadas com materiais de hidrogel, que contêm baixo, médio ou alto conteúdo de água. LCG tóricas com baixo teor de água funcionam bem do ponto de vista fisiológico, se forem finas. No entanto, devido ao grau e ao método de estabilização, a maioria tornase espessa, exigindo um material com conteúdo aquoso médio ou alto para garantir à córnea a transmissibilidade de $\mathrm{O}_{2}$ suficiente para manter sua fisiologia dentro dos padrões de normalidade. De modo geral, recomenda-se não dormir com esse tipo de LC.

Estão disponíveis em graus esféricos de -20,0 D a +20,0 D; graus cilíndricos de $-0,75 \mathrm{D}$ a $-10,0 \mathrm{D}$, com eixos de rotação em incrementos de $5^{\circ}$ ou até de $1^{\circ}$.

\section{LCG Tóricas Descartáveis / Troca Programada}

O desenvolvimento de LCG tóricas para esquemas de descartabilidade tornou mais fácil passar o usuário de LCG esférica para a LCG tórica quando existe um astigmatismo comprometendo o desempenho visual. As vantagens são as mesmas das descartáveis esféricas.

A relação das LCG disponíveis no Brasil, até 2000, está em "O Consultor" (SOBLEC) e nos EUA em Contact Lenses Quarterly.

\section{REFERÊNCIAS}

1. Holden BA. The principles and practice of correcting astigmatism with soft contact lenses. Aust J Optom 1975;58:279-99

2. Lindsay RG, Westerhout DI. Toric contact lens fitting. In.: Phillips AJ \& Speedwel L. Contact Lenses. $4^{\text {th }}$ ed. Rochester, Kent: Butterworth-Heinemann; 1997. p. 464-93.

3. Tomlinson A, Bibby MM. Lid interaction and toric soft lens axis location Am J Optom Physiol Opt 1982;59:228-33.

4. Gundel RE. Effect of cylinder axis on rotation for a double thin zone design toric hydrogel. Int Contact Lens Clin 1989;16:141-45.

5. Coral-Ghanem C, Stein HA, Freeman MI. Fundamentos e adaptação das lentes de contato hidrofilicas $(\mathrm{LCH})$. In: Coral-Ghanem C, Stein HA, Freeman M. Lentes de contato do básico ao avançado 2000. Joinville: Soluções e Informática; 2000. p. 25-40. 